hep-th/9302021

\title{
SU(2) and the Kauffman bracket
}

\author{
B Broda $\dagger$ \\ Institute for Mathematical Physics, Technical University of Clausthal, Leibnizstraße 10, \\ D-W-3392 Clausthal-Zellerfeld, Federal Republic of Germany
}

A direct relationship between the (non-quantum) group SU(2) and the Kauffman bracket in the framework of Chern-Simons theory is explicitly shown.

$\dagger$ Permanent address: Department of Theoretical Physics, University of Łódź, Pomorska 149/153, PL-90-236 Łódź, Poland; e-mail: ptbb@ibm.rz.tu-clausthal.de 
In his seminal paper on quantum field theory and the Jones polynomial [1], Edward Witten proposed, in the framework of Chern-Simons theory based on the group SU(2), a new approach to invariants of knots and links. In this letter, we would like to show that the mathematical object directly related to the fundamental representation of $\mathrm{SU}(2)$ is the Kauffman bracket [2]. Strictly speaking, we will show that SU(2) Chern-Simons theory at the level $k$, with line observables defined in the fundamental representation, directly corresponds to the (one-variable/specialization of the) Kauffman bracket (regular isotopy invariant of unoriented links) with the parameter $A=\exp (-\pi i / 4 k)$.

The "half-monodromy" or (quasi-)braiding matrix derived from the Chern-Simons theory is of the form $[3-4]$

$$
\mathbb{B}=\exp \left(-\frac{\pi i}{k} \mathbf{t}^{\otimes 2}\right), \quad k \in \mathbf{Z}^{ \pm}
$$

where $\mathbf{t}$ is a generator of a compact semi-simple Lie group $G$. Putting for $G=\mathrm{SU}(2)$ $t^{a}=\frac{1}{2} \sigma^{a}, a=1,2,3$ ( $\sigma^{\prime}$ s are the Pauli matrices), and using the Fierz identity, we obtain

$$
\begin{aligned}
\mathbb{B} & =\exp \left(-\frac{\pi i}{4 k} \sum_{a=1}^{3} \sigma_{i j}^{a} \otimes \sigma_{k l}^{a}\right) \\
& =\exp \left[\frac{\pi i}{4 k}(\mathbb{I}-2 \mathbb{E})\right] \\
& =\exp \left(\frac{\pi i}{4 k}\right)\left(\mathbb{I} \cos \frac{\pi}{2 k}-i \mathbb{E} \sin \frac{\pi}{2 k}\right),
\end{aligned}
$$

with

$$
\mathbb{I} \equiv \delta_{i j} \delta_{k l}, \quad \mathbb{E} \equiv \delta_{i l} \delta_{k j}
$$

The correspondence

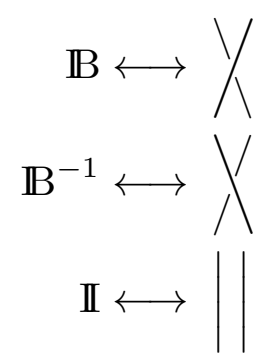


yields the following skein relation

$$
\left.A\langle\bigvee\rangle-A^{-1}\langle\rangle\right\rangle=\left(A^{2}-A^{-2}\right)\langle||\rangle,
$$

where

$$
A=\exp \left(-\frac{\pi i}{4 k}\right)
$$

and " \langle\rangle " denotes the normalized quantum-field-theory expectation value with respect to the Chern-Simons "measure". Rotating the graphs in (5), we obtain a new equivalent skein relation

$$
A\langle\rangle\rangle-A^{-1}\langle\bigvee\rangle=\left(A^{2}-A^{-2}\right)\left\langle\begin{array}{l}
U \\
\bigcap
\end{array}\right\rangle
$$

Combining (5) and (7) produces

$$
\langle\backslash\rangle=A\left\langle\begin{array}{l}
U \\
\bigcap
\end{array}\right\rangle+A^{-1}\langle||\rangle
$$

All the lines entering our graphs should be unoriented. It follows from the fact that the fundamental representation of the group $\mathrm{SU}(2)$ is non-complex (pseudo-real) [5], and the expectation values of the line observables in the fundamental representation have to be invariant with respect to the reversing of orientation [4].

To compute the dependence of a line on a framing one should contract two indices in the exponent of $\mathbb{B}$ (say, $j$ and $k$ ) yielding

$$
\exp \left(-\frac{3 \pi i}{4 k}\right)=A^{3}
$$

Thus,

$$
\langle\mid \pm 1\rangle=-A^{ \pm 3}\langle\mid 0\rangle
$$

where the minus sign follows from the pseudo-reality of the fundamental representation of $\mathrm{SU}(2)$, and the integers mean the framing. Closing in (8) the left legs of all the (three) diagrams with arcs, as well as the right ones, and applying (10), we obtain

$$
\langle\bigcirc\rangle=-A^{2}-A^{-2} .
$$


In (11), we have used the property of locality of Chern-Simons theory, which can be expressed as

$$
\left\langle L_{1} \sqcup L_{2}\right\rangle=\left\langle L_{1}\right\rangle\left\langle L_{2}\right\rangle
$$

where the symbol "ப" denotes a distant union of links (links separated with a twosphere).

Collecting equations (8), (11) and (12) we can write down the full set of the axioms of the (one-variable) Kauffman bracket:

(i)

$$
\langle\emptyset\rangle=1
$$

(ii)

$$
\langle\bigcirc \sqcup L\rangle=\left(-A^{2}-A^{-2}\right)\langle L\rangle
$$

(iii)

$$
\langle\rangle\rangle=A\left\langle\begin{array}{l}
\cup \\
\bigcap
\end{array}\right\rangle+A^{-1}\langle\|\rangle,
$$

where $A=\exp (-\pi i / 4 k)$.

\section{Acknowledgments}

The author is indebted to Professor H. D. Doebner for his kind hospitality in Clausthal. The work was supported by the Alexander von Humboldt Foundation and the KBN grant 202189101. 


\section{References}

[1] Witten E 1989 Commun. Math. Phys. 121351

[2] Kauffman L H 1987 Topology 26395

Kauffman L H 1991 Knots and Physics (World Scientific)

[3] Broda B 1990 Mod. Phys. Lett. A 52747

[4] Guadagnini E 1992 Int. J. Mod. Phys. A 7877

[5] O'Raifeartaigh L 1988 Group Structure of Group Theories (Cambridge: CUP) p 57 